\title{
Universiteit
}

Leiden

The Netherlands

\section{Physical properties and microstructure of ultrathin (La,Ca) MnO3 films under different conditions of strain}

Yang, Z.-Q.; Hendrikx, R.W.A.; Aarts, J.; Qin, Y.L.; Zandbergen, H.W.

\section{Citation}

Yang, Z. -Q., Hendrikx, R. W. A., Aarts, J., Qin, Y. L., \& Zandbergen, H. W. (2004). Physical properties and microstructure of ultrathin ( $\mathrm{La}, \mathrm{Ca}) \mathrm{MnO} 3$ films under different conditions of strain. Physical Review B, 70(17), 174111. doi:10.1103/PhysRevB.70.174111

Version: $\quad$ Not Applicable (or Unknown)

License: $\quad$ Leiden University Non-exclusive license

Downloaded from: https://hdl.handle.net/1887/45423

Note: To cite this publication please use the final published version (if applicable). 


\title{
Properties and microstructure of ultrathin $(\mathrm{La}, \mathrm{Ca}) \mathrm{MnO}_{3}$ films under different conditions of strain
}

\author{
Z. Q. Yang, R. Hendrikx, and J. Aarts \\ Kamerlingh Onnes Laboratory, Leiden University, P.O. Box 9504, 2300 RA Leiden, The Netherlands \\ Y. L. Qin* and H. W. Zandbergen \\ National Center for High-resolution Electron Microscopy, Laboratory of Materials Science, Delft University of Technology, \\ Rotterdamseweg 137, 2628 Al Delft, The Netherlands
}

(Received 17 January 2004; published 22 November 2004)

\begin{abstract}
Ultrathin films of $\mathrm{La}_{1-x} \mathrm{Ca}_{x} \mathrm{MnO}_{3}(x \cong 0.33)$ in a thickness range below $10 \mathrm{~nm}$ were deposited by direct current (dc) magnetron sputtering on different substrates, (001) $\mathrm{SrTiO}_{3}$ (STO), (001) $\mathrm{LaAlO}_{3}$ (LAO), and (110) $\mathrm{NdGaO}_{3}$ (NGO) in order to study the effects of strain in this thickness regime. The combined study of physical properties and microstructure by high-resolution electron microscopy (HREM) reveals that ultrathin $(\$ 6 \mathrm{~nm})$ strained films on STO can be grown in a body-centered-tetragonal structure not recognized previously. Such films do not show an insulator-metal (IM) transition. At slightly larger thickness, around $6 \mathrm{~nm}$, the microstructure is different. Depending on the growth parameters we find either a structure involving $\mathrm{MnO}_{6}$-octahedra tilted around two axes, in combination with the occurrence of twin boundaries as reported previously; or the bulk orthorhombic Pnma structure. In the latter case an IM transition is found at temperatures close to the bulk value. On lattice-matched NGO, the IM transition and bulk-like magnetoresistance effects are observed down to the lowest thickness investigated $(3 \mathrm{~nm})$. On LAO, which compresses the lattice, no IM transition is found even at a thickness of $15 \mathrm{~nm}$, which is due to island-like growth, as confirmed by HREM observations.
\end{abstract}

DOI: 10.1103/PhysRevB.70.174111

PACS number(s): 68.55.Jk, 72.15.Gd, 81.15.Cd

\section{INTRODUCTION}

After the discovery of the colossal magnetoresistance (CMR) effect, ${ }^{1}$ the mixed-valent manganites $\mathrm{A}_{1-x} \mathrm{~B}_{x} \mathrm{MnO}_{3}$ with $\mathrm{A}$, a trivalent rare earth ion such as $\mathrm{La}, \mathrm{Pr}$, or $\mathrm{Nd}$, and $\mathrm{B}$, a divalent alkaline ion such as $\mathrm{Ca}, \mathrm{Sr}$, or $\mathrm{Ba}$, have attracted renewed interest. They show fascinating physical phenomena ${ }^{2}$ as well as technological potential, for instance in the field of spin electronics. ${ }^{3}$ Their properties basically derive from the fact that doping the parent compound $\mathrm{AMnO}_{3}$ with divalent $\mathrm{B}$ atoms leads to a mixture of $\mathrm{Mn}^{3+}$ and $\mathrm{Mn}^{4+}$ ions. This yields an increased hopping probability for the (doped) carriers, which leads to a ferromagnetic ground state through the so-called double-exchange mechanism. ${ }^{4,5}$ In the CMR manganites, the high temperature state is paramagnetic and insulating, but increased ferromagnetic interactions when lowering the temperature invoke a transition to the ferromagnetic metallic state, which is therefore basically a magnetically driven insulator-to-metal (IM) transition. Due to the fact that the ferromagnetic interactions allow only one spin species to hop, such materials essentially show a spin polarization of $100 \% .^{6}$ Also, the formation of the metallic band is in competition with an electron-lattice coupling on the Mn sites, which tends to localize electrons by the Jahn-Teller effect through a deformation of the oxygen octahedra surrounding the $\mathrm{Mn}$ atom. ${ }^{7}$

The $\mathrm{ABO}_{3}$-type manganite oxides have a pseudocubic perovskite structure which involves a network of cornersharing $\mathrm{MnO}_{6}$ octahedra, and it follows that, at constant doping, important properties such as the temperature of the IMtransition $T_{\mathrm{IM}}$ still sensitively depend on the bond angles and distances within this network. One way to influence these is by varying the $\mathrm{A}$, B-cation radii with respect to the $\mathrm{O}$-anion radius. Starting from an ideal simple cubic perovskite, the structure will change in order to accommodate such a nonoptimal A or B ion, for instance by distorting or tilting the octahedra. This leads to a large number of closely related structures with different spacegroups. ${ }^{8-10}$ Also, within one structure the amount of deformation can vary, leading to the well-known tuneability of the physical properties. ${ }^{11-13}$

Another way to influence structure and properties is by growing thin films on nonmatched substrates. Given the number of possible structures, it might be expected that the strain induced by a substrate with lattice parameter different from the material of the film can also lead to variations of properties and structure. Such strain engineering is of fundamental interest as well as of practical importance for the development of electronic and magnetic devices using thin films. However, additional factors now play a role since the electronic properties are also sensitive to, e.g., growthinduced disorder, oxygen inhomogeneities, and obviously strain relaxation. For instance, it was recently shown that inhomogeneously released strain can lead to the occurrence of phase separation: in films of $\mathrm{La}_{0.67} \mathrm{Ca}_{0.33} \mathrm{MnO}_{3}$ on $\mathrm{LaAlO}_{3}$ (compressive strain) coexistence was found of a ferromagnetic metallic state in low strain regions and a chargeordered insulator in high strain regions. ${ }^{14,15}$ Moreover, also the deposition method and growth parameters are important, since they determine the growth mode of the film and therefore the strain state.

In this paper we focus on the question of strain and strain relaxation in the archetypical CMR material 
$\mathrm{La}_{0.67} \mathrm{Ca}_{0.33} \mathrm{MnO}_{3}$ (LCMO) by studying the microstructure and properties of films deposited by high oxygen pressure magnetron sputtering at very low film thickness $d_{L}$ $(<10 \mathrm{~nm})$. We use various substrates but mainly discuss the case of tensile strain produced by the larger lattice parameter of (001) $\mathrm{SrTiO}_{3}$ (STO) where the most interesting effects are observed. Bulk LCMO has a Pnma orthorhombic structure ${ }^{13}$ in which the $\mathrm{MnO}_{6}$ octahedra are tilted around all three independent cartesian axes. The unit cell of the structure is enlarged with respect to the cubic cell of size $a_{p}$, and has unit vectors very close to $\sqrt{ } 2 a_{p}, 2 a_{p} \sqrt{ } 2 a_{p}$. The lattice parameter of the structure given in terms of the $a_{p}$ equals $0.386 \mathrm{~nm}$. In previous work it was shown that for thick films $\left(d_{L}\right.$ between 10 and $200 \mathrm{~nm})$ deposited on STO $\left(a_{p}=0.391 \mathrm{~nm}\right)$ the film structure is also Pnma, with the (doubled) $b$ axis out-ofplane. The enlarged in-plane lattice parameter leads to a smaller out-of-plane value which only slowly relaxes back to the bulk value with increasing thickness. ${ }^{16}$ For such strained films, $T_{\mathrm{IM}}$ is clearly lower than the bulk value of about $270 \mathrm{~K} \cdot{ }^{16,17}$ For smaller thicknesses, around $6 \mathrm{~nm}$, a different structure was observed. It is a two-tilt structure with the tilt around the $b$ axis missing, and distortions of the oxygen squares in the $a, c$ plane combined with a regular twin structure. ${ }^{18}$ In the present work, we go to even smaller thickness, and discover that around $3 \mathrm{~nm}$ the structure is still different. It has modified into a one-tilt body-centeredtetragonal (bct) structure with space group $I 4 / \mathrm{mcm}$, which is ferromagnetic below $150 \mathrm{~K}$, but insulating, and does not show an IM transition down to $5 \mathrm{~K}$. Apparently, the critical thickness for this fully strained structure lies at about $5 \mathrm{~nm}$ : around this thickness, both the one-tilt and the two-tilt structures can be found. However, this also depends on the sputter current: a higher current and the ensuing faster growth can evoke the bulk Pnma orthorhombic structure, with a bulklike $T_{\mathrm{IM}}$. The bct structure is clearly due to the substrate strain. We also deposited ultrathin films on lattice-matched substrates of $(110) \mathrm{NdGaO}_{3}(\mathrm{NGO})\left(a_{p}=0.386 \mathrm{~nm}\right)$ and (001) $\mathrm{LaAlO}_{3}(\mathrm{LAO})\left(a_{p}=0.379 \mathrm{~nm}\right)$. On NGO, films down to $3 \mathrm{~nm}$ are of the bulk Pnma orthorhombic structure, with a bulk-like IM transition. The depression of $T_{\mathrm{IM}}$ appears due to growth-related disorder and involvement of small amounts of a simple cubic phase. On LAO with compressive strain in the substrate plane, no continuous films, but rather an island-like structure is grown at thicknesses up to $15 \mathrm{~nm}$, and consequently no IM transition is observed.

\section{EXPERIMENT}

All films were magnetron-sputter deposited from ceramic targets of $\mathrm{La}_{1-x} \mathrm{Ca}_{x} \mathrm{MnO}_{3}$ with a nominal composition of $x$ $\sim 0.33$ on single crystalline STO, LAO, and NGO substrates of $10 \mathrm{~mm}$ by $10 \mathrm{~mm}$, in a pure oxygen atmosphere of $300 \mathrm{~Pa}$. The high pressure leads to a very low growth rate. We used two different sputter currents, leading to two different rates of 1.4 and $0.8 \mathrm{~nm} / \mathrm{min}$, denoted as fast and slow growth, respectively. The growth temperature was chosen at $840{ }^{\circ} \mathrm{C}$. After deposition, the sample chamber was quickly evacuated and the grown films were cooled to room temperature at a rate of $100{ }^{\circ} \mathrm{C} / \mathrm{min}$ without further post-annealing.
The films were characterized by x-ray diffraction and highresolution electron microscopy (HREM). Cross-section specimens for HREM were mechanically ground by tripod directly down to a homogeneous thickness less than $10 \mu \mathrm{m}$. During ion milling, the specimen was not rotated and was oriented such that the thin-film side was facing away from ion gun. More details of the sample preparation were published elsewhere. ${ }^{19}$ HREM and electron diffraction (ED) were performed with a Philips CM 30UT electron microscope with a field emission gun operated at $300 \mathrm{kV}$. A condenser aperture of $10 \mu \mathrm{m}$ and a small spot size of $5 \mathrm{~nm}$ were used to obtain ED patterns of the ultrathin films. In specifying the viewing direction in the HREM experiments, we will generally use the substrate direction; viewing along the [001] direction of STO is denoted as $[001]_{\text {STO }}$. Note that $[001]_{\text {STO }}$

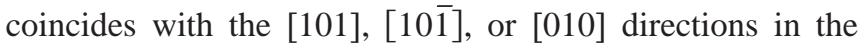
orthorhombic unit cell of LCMO. Transport measurements were performed on unstructured LCMO films using a commercial measurement platform. For this, the substrates were cut in two halves and four Au contacts were sputtered onto the film. The distance between the voltage contacts was about $3 \mathrm{~mm}$. Magnetization was measured with a commercial "superconducting quantum interference device" magnetometer.

\section{RESULTS AND DISCUSSION}

To study the role of strain on the initial growth of the films, we first deposited $\mathrm{La}_{0.67} \mathrm{Ca}_{0.33} \mathrm{MnO}_{3}$ films of 6 and $3 \mathrm{~nm}$ on NGO in fast growth mode. The resistance $R$ as function of temperature $T$ is plotted in Figs. 1(a) and 1(b). It is clear that ultrathin films on lattice matched NGO show bulk-like properties, i.e., an IM transition and large magnetoresistance (MR) effects. For the 6-nm film $T_{\mathrm{IM}} \approx 215 \mathrm{~K}$. Some influence of disorder is apparently present, because in films of $50 \mathrm{~nm}, T_{\mathrm{IM}} \approx 270 \mathrm{~K}$, the bulk value. ${ }^{16}$ For the 3-nm film the IM transition is still present, although $T_{\mathrm{IM}}$ now drops to $125 \mathrm{~K}$. Note that at room temperature the value of $R \times d_{L}$ only differs by a factor of about 2 , which shows that these films can be grown quite homogeneously at small thickness.

The fact that the films are smooth and epitaxial is observed in HREM. The NGO substrate has the Pnma orthorhombic structure, the same as bulk LCMO. The $(001)_{\mathrm{NGO}}$ surface plane is therefore equivalent to $(110)_{\text {STO }}$. Shown in Fig. 2 is a HREM picture of the $3-\mathrm{nm}$ film on NGO, viewed along $[100]_{\mathrm{NGO}}$. In this case, the LCMO grows coherently on NGO, except for some defects at the interface, one of which is marked by an arrowhead. The film consists of mostly Pnma bulk structure with both [001]-type superstructure (left side of the picture) and [100]-type superstructure (not shown), while in some small area (right side) a simple cubic phase is found. HREM images of the $6 \mathrm{~nm}$ film on NGO show that, for this thicker film, the most part has the bulk Pnma structure, with a [100]-type superstructure which just follows the NGO substrate. In general, there are more areas with bulk structure in 6-nm film than in the 3-nm film, and most of them have a single orientational relationship with NGO. The (doubled) $b$ axis is found parallel to the interface, 


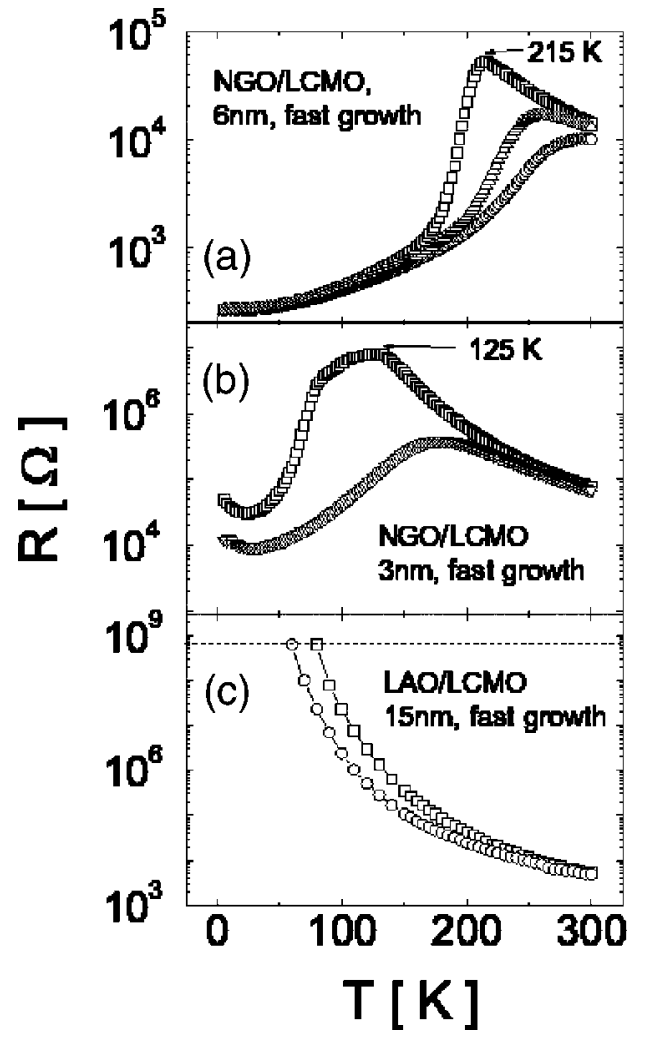

FIG. 1. Resistance as function of temperature measured at applied fields $\mu_{0} H_{a}$ of $0 \mathrm{~T}$ (squares), $4 \mathrm{~T}$ (triangles), and $8 \mathrm{~T}$ (open circles) for films of $\mathrm{La}_{0.67} \mathrm{Ca}_{0.33} \mathrm{MnO}_{3}$ deposited in fast growth mode. (a) $6 \mathrm{~nm}$ on NGO; (b) $3 \mathrm{~nm}$ on NGO; (c) $15 \mathrm{~nm}$ on LAO. The dashed line in Fig. 1(c) denotes the limit of the resistance measurement.

which we always find to be the case for films on NGO. This is probably due to the fact that the bulk LCMO $b$-axis lattice parameter $b_{p}=0.38615 \mathrm{~nm}$ matches the substrate slightly better than the $a, c$ axes $\left(a_{p}=0.38646 \mathrm{~nm}, c_{p}\right.$ $=0.38674 \mathrm{~nm}) .^{20}$ Therefore, on lattice-matched NGO, epitaxial films can be grown down to $3 \mathrm{~nm}$ with bulk structure. The observed decrease of $T_{\mathrm{IM}}$ in this thickness range, which is commonly observed, ${ }^{21}$ appears to be due to small amounts of a simple cubic structure rather than to other types of disorder such as twinning.

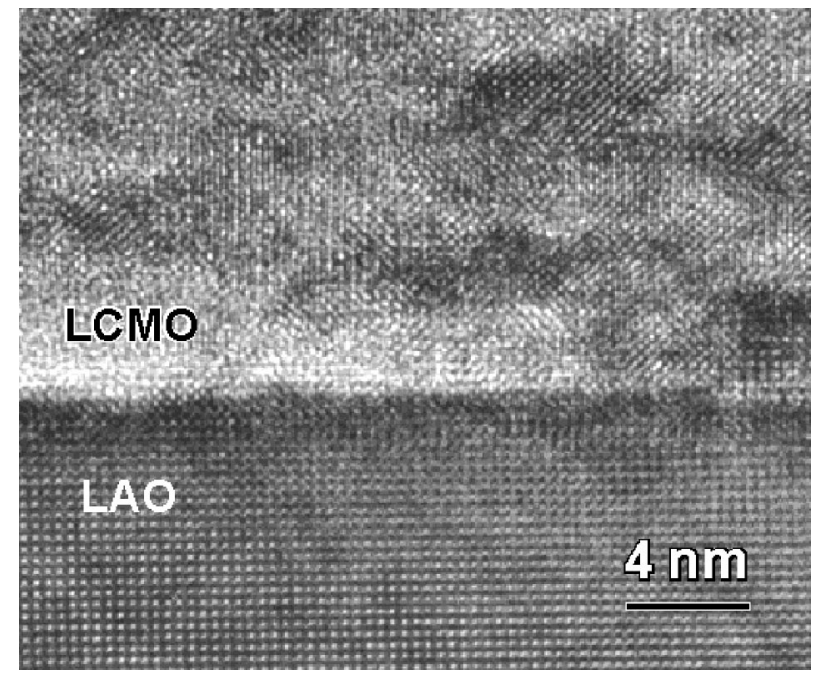

FIG. 3. HREM image of a $15 \mathrm{~nm}$ LCMO film on LAO viewed along $[100]_{\text {LAO }}$ : the bright area is amorphous, some smaller areas with darker contrast are polycrystalline.

A plot of $R(T)$ for a $15 \mathrm{~nm}$ film on LAO with compressed in-plane lattice parameter is given in Fig. 1(c). $R$ increases with decreasing $T$. Below $100 \mathrm{~K}$, where the measurement limit of $10^{9} \Omega$ is reached, no return to lower resistance values was found down to $5 \mathrm{~K}$, which indicates the absence of an IM transition. The microstructure of films grown on LAO is shown in Fig. 3. It is polycrystalline and in some areas is even amorphous. In this case the disorder precludes an IM transition and MR effects. On larger scales, an island-like structure is found, and thicker films usually show strong columnar features. ${ }^{22}$ It is worth noting that different results are reported for the same or similar substrate/film combinations when the films are grown by pulsed laser deposition. ${ }^{21,23}$ These differences between the two deposition techniques have not been fully explored yet.

Next we turn to the films on (tensile) STO. As reported before, ${ }^{16,18}$ these films are pseudomorphic and epitaxial. $\mathrm{X}$-ray diffraction using the (002) and (103) reflections shows values of the in-plane lattice parameter at the substrate value of $3.91 \mathrm{~nm}$, and of the out-of-plane lattice parameter around $3.825 \mathrm{~nm}$.

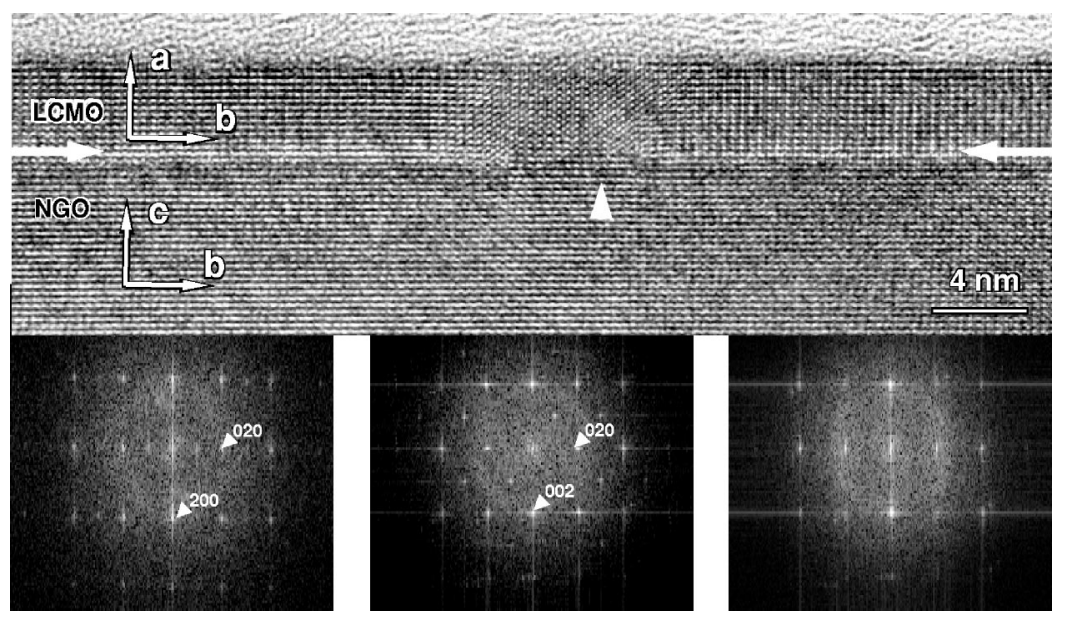

FIG. 2. HREM image (upper) and Fourier transform (FT) patterns (lower) of a $3 \mathrm{~nm} \mathrm{LCMO}$ film on NGO viewed along $[100]_{\mathrm{NGO}}$. The horizontal white arrows mark the position of the interface. The verical white arrow points to a defect. FT patterns (left) taken from the left part of the LCMO film and showing a [001]-type superstructure; (middle) taken from the substrate and showing a [100]-type superstructure; (right) taken from the right part of the LCMO film and showing no superstructure (simple cubic). 


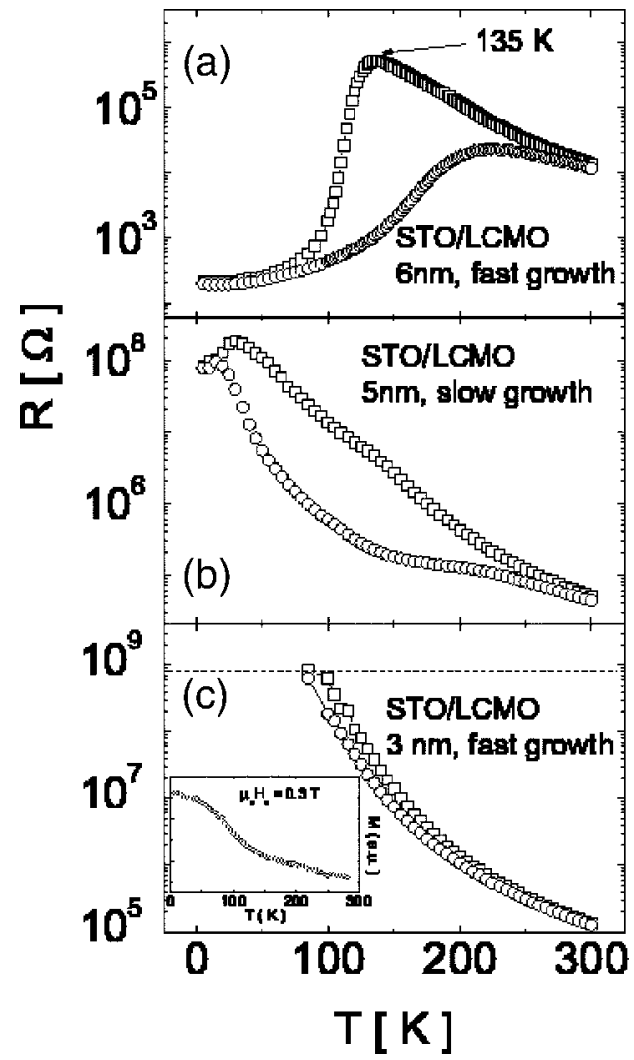

FIG. 4. Resistance as function of temperature in applied field $\mu_{0} H_{a}$ of $0 \mathrm{~T}$ (squares) and $8 \mathrm{~T}$ (open circles) for $\mathrm{La}_{0.67} \mathrm{Ca}_{0.33} \mathrm{MnO}_{3}$ films of different thicknesses deposited on STO using different growth rates: (a) fast growth, $6 \mathrm{~nm}$; (b) slow growth, $5 \mathrm{~nm}$; (c) fast growth, $3 \mathrm{~nm}$. The inset in (c) shows the temperature dependent magnetization $M$ at $\mu_{0} H_{a}=0.3 \mathrm{~T}$ for the $3-\mathrm{nm}$ film.

Figure 4 shows $R(T)$ for films grown on STO with thickness $3 \mathrm{~nm}$ (fast growth), $5 \mathrm{~nm}$ (slow growth), and $6 \mathrm{~nm}$ (fast growth). The fast growth film of $6 \mathrm{~nm}$ shows an IM transition at $135 \mathrm{~K}$ [Fig. 4(a)]. The $5 \mathrm{~nm}$ slow growth film is an insulator down to $4 \mathrm{~K}$ without showing an IM transition [Fig. 4(b)] but with a large MR effect in a field of $8 \mathrm{~T}$. This behavior is the same as we reported previously ${ }^{17,18}$ for films around $6 \mathrm{~nm}$ which were grown at a rate of $0.9 \mathrm{~nm} / \mathrm{min}$, very close to the rate we now call "slow growth." Fast growth therefore appears to favor a microstructure which al- lows the IM transition. However, even for fast growth, $R(T)$ of the $3 \mathrm{~nm}$ film shows insulating behavior without MR effects [Fig. 5(c)], while in the magnetization [inset in Fig. 4(c)] onset of ferromagnetism is observed around $150 \mathrm{~K}$.

To explain these differences, we again turn to the structural information. HREM images (not shown) were taken of a fast grown 6-nm LCMO film viewed parallel to the interface and along $[100]_{\text {Sто }}$ (equal to [101] of LCMO). By tilting the specimen from the exact zone axis over about $1^{\circ}$, the usual $2 a_{p}$ fringes appeared. ${ }^{24}$ Defects, which show up as a shift of the $2 a_{p}$ fringes over a distance $a_{p}$ were frequently observed throughout the investigated area. They look very similar to the defects seen in the earlier slow-growth films, ${ }^{25}$ which were explained as twin boundaries (TBs) where the $a$ and $c$ directions are interchanged. If this is the case, it is expected that images taken along [110 $]_{\text {STO }}$ should demonstrate both [100]- and [001]-oriented LCMO. These can be easily distinguished since the [100] direction has $n$-glide mirror symmetry and the [001] direction has $a$-glide mirror symmetry. Figure 5(a) shows a HREM image of the $6 \mathrm{~nm}$ fast growth film viewed along $[110]_{\text {STO }}$. Both the [100] and [001] orientations are found, which can be clearly seen in the enlarged images shown in Figs. 5(b) and 5(c), respectively. This is quite different from the previous work. In that case, [100]-type images could be found regularly, but [001]-type images were fully absent, which could only be explained by assuming a structure where rotations of the $\mathrm{MnO}_{6}$-octahedra around the $b$ axis are absent (two-tilt structure). The conclusion now is that this structure only occurs under slow-growth conditions. Fast growth still leads to $a, c$ twinning in the plane of the substrate, but with the bulk (three-tilt) structure. In very nice correlation with this, the physical properties of such films are bulk-like, with strong MR effects and a relatively high value of $T_{\mathrm{IM}}[135 \mathrm{~K}$ for the film shown in Fig. 4(a)]. The reduction with respect to the bulk value can then be attributed to residual strain. . $^{16-26-28}$

For fast-growth films thinner than $6 \mathrm{~nm}$, in particular the 3-nm film shown in Fig. 4(c), neither the $2 a_{p}$ fringes nor the TBs were observed. As is shown in Fig. 6, this film exhibits a perfect single crystalline structure and grows coherently on the STO substrate. Absence of the $2 a_{p}$ fringes [see Fig. 6(a), where the image is taken along [100 $]_{\text {Sто }}$, indicates that the crystalline structure of this film is not the Pnma orthorhombic, but more likely the one-tilt $14 / \mathrm{mcm}$ body-centered-

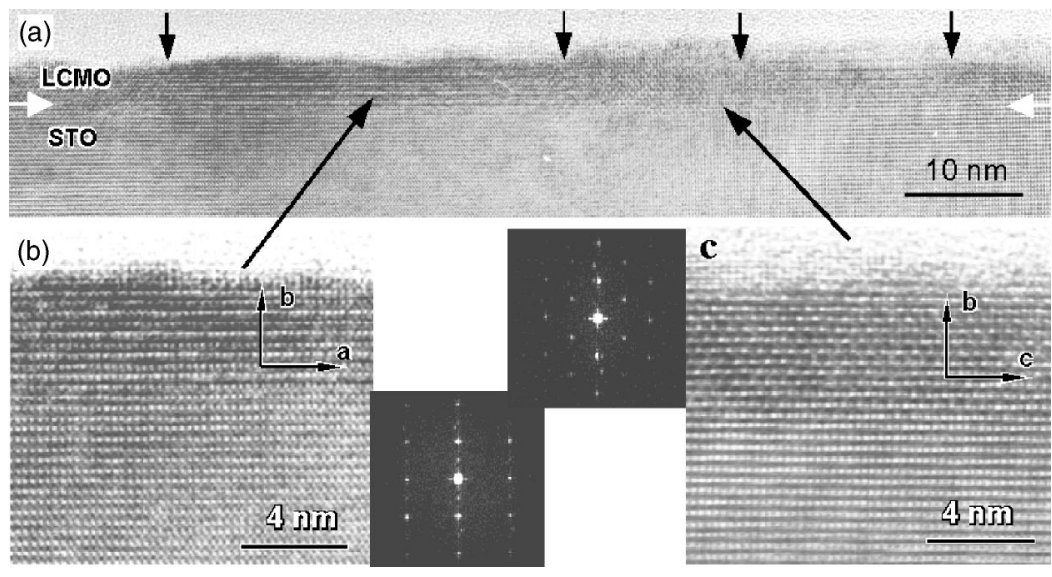

FIG. 5. HREM image of a $6 \mathrm{~nm}$ fast growth LCMO film on STO viewed along $[110]_{\text {STO }}$ (a) Overview; the white arrowhead marks the interface. Both the [100]- and [001]-type superstructures are observed throughout the film. The upper arrows define the enlarged areas. (b), (c) Two enlarged areas of the overview together with their FT pattern. In (b) the [001]-type superstructure is present; (c) shows the [100]-type superstructure. 

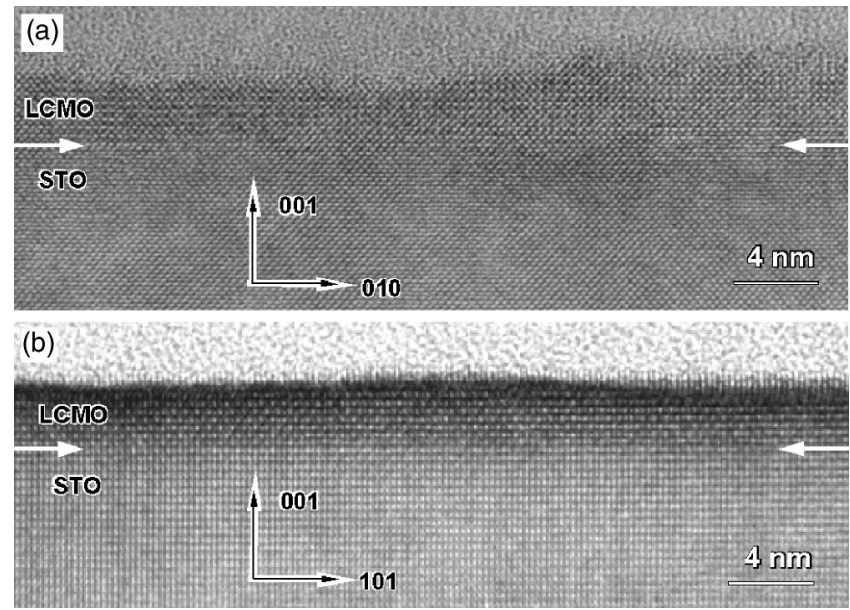

FIG. 6. Cross-sectional HREM images of a $3 \mathrm{~nm}$ LCMO film on STO taken along (a) $[100]_{\text {STO }}$ and (b) $[110]_{\text {STO }}$ directions, respectively.

tetragonal $a_{p} \sqrt{ } 2, a_{p} \sqrt{ } 2,2 a_{p}$ structure. This is confirmed by the image taken along $[110]_{\text {Sто }}$ [Fig. 6(b)] where also only a single structure is observed. The details of this structure will be published elsewhere. ${ }^{29}$ HREM observation on the slow growth 5-nm film (not shown here) also showed the tetragonal structure rather than either the Pnma orthorhombic bulk structure or the "thin-film" two-tilt structure.

The full description for growth of $\mathrm{La}_{0.67} \mathrm{Ca}_{0.33} \mathrm{MnO}_{3}$ on $\mathrm{SrTiO}_{3}$ under tensile strain therefore includes the growth rate as parameter in determining what structure is formed during cooling down. It should be noted that the parameter which is actually changed is the sputter current. It cannot be excluded that it is not the growth rate which is determining factor, but another one which influences the mobility of atoms on the growing surface, for instance the plasma potential. Still, we will continue to use the growth rate for descriptive purposes, and we can put all results together in the structural phase diagram given in Fig. 7.

The thinnest, most strained films $(3 \mathrm{~nm}$ fast and $5 \mathrm{~nm}$ slow) show a one-tilt body-centered-tetragonal structure and insulating behavior, sometimes with MR effects. The MR effects may be due to involvement of the orthorhombic phase. Slightly thicker films have a different structure. Fast growth yields the three-tilt bulk Pnma orthorhombic structure with $a-c$ twinning, but always with the $b$ axis out of the substrate plane. The critical thickness $d_{\text {cr }}$ for this structural difference lies around $6 \mathrm{~nm}$. Also, close to $d_{\mathrm{cr}}$, slow growth yields the thin-film two-tilt structure, although this now appears a somewhat fragile or metastable one. Films around $d_{\text {cr }}$ show mixed physical properties. In four more 6-nm slow growth films, one showed MR effects and an IM transition, the other three showed MR effects but no IM transition. The MR effects may well be due to the two-tilt structure, but a mixture of the orthorhombic and tetragonal structures cannot be ruled out. Increasing the thickness, for both growth modes, leads to a gradual change to the bulk three-tilt structure. The $b$ axis now can have both in-plane and out-of-plane directions. The tetragonal structure, finally, appears to be ferromagnetic insulating (FI). As can be seen from the inset in Fig. 4(c), onset of ferromagnetism is found at about $150 \mathrm{~K}$. Due to the substrate contribution, it is difficult to estimate the saturation moment, but comparison with values from $6 \mathrm{~nm}$ films indicate that the moment per $\mathrm{Mn}$ ion is roughly similar, of the order of $2 \mu_{B} / \mathrm{Mn}$. Such reduction in moment has been ascribed to the onset of orbital ordering. ${ }^{30} \mathrm{~A}$ full crossover to an antiferromagnetic state may even be expected below a certain $c / a$ ratio. ${ }^{31}$ In our case, $c / a \approx 0.97$, which is apparently not enough to invoke full orbital ordering and antiferromagnetism. On the other hand, the compression should invoke Jahn-Teller-like deformations along the out-of-plane direction, and the ensuing localization effects are probably responsible for the insulating state. Thin strained LCMO layers could therefore be used in FI tunnel junctions.

\section{CONCLUSION}

In conclusion, we have studied the physical properties and microstructure of ultrathin films of $\mathrm{La}_{0.67} \mathrm{Ca}_{0.33} \mathrm{MnO}_{3}$ on substrates with different lattice mismatch. The results emphasize the large differences which can be invoked in the physical properties of these materials when biaxial strain is applied, in particular in the regime of very thin films. On lattice matched $\mathrm{NdGaO}_{3}$, films down to $3 \mathrm{~nm}$ with bulk Pnma orthorhombic structure show the IM transition and CMR albeit with a reduced value for $T_{\mathrm{IM}}$ due to growth related disorder. On compressive $\mathrm{LaAlO}_{3}$, no epitaxial film is grown due to island growth mode; at least for sputtering deposition, the orthorhombic bulk structure is amenable to growth of an expanded or matched lattice, but growth of a compressed lattice is inhibited. This once more emphasizes the differences between deposition by magnetron sputter and pulsed laser ablation. On applying tensile strain using $\mathrm{SrTiO}_{3}$, we find that

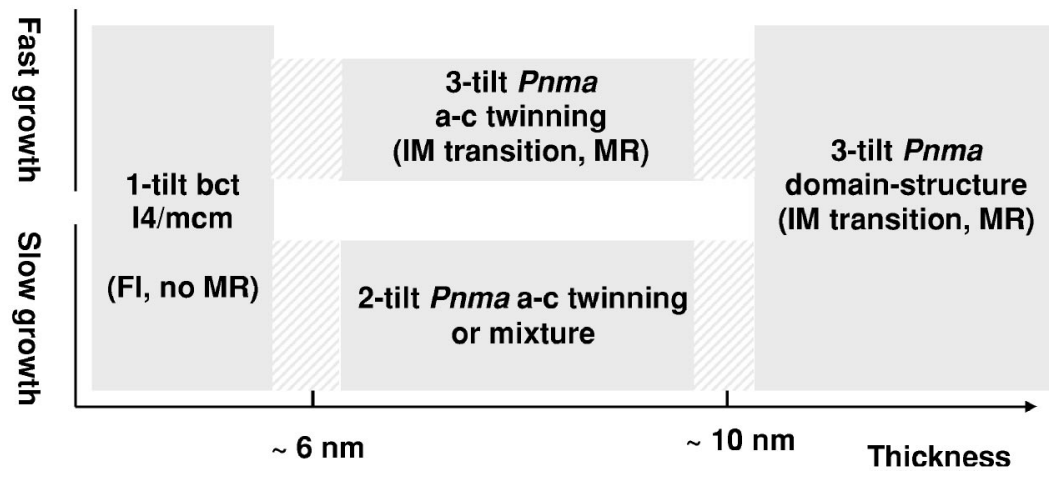

FIG. 7. Phase diagram for the different crystal structures with their fundamental physical properties identified for strained LCMO films on STO as function of thickness, for two different growth rates. 
strain not only changes the film properties in a quantitative manner, meaning a lowering of $T_{\mathrm{IM}}$; for films below a critical thickness of about $5 \mathrm{~nm}$ we find a qualitative change, namely a different crystal structure with different physical properties (ferromagnetic insulating). This should be kept in mind when using strain to engineer specific properties. Moreover, since this case of LCMO on STO until now provides the only observation of a fully different structure at small film thickness, it opens the question how general the phenomenon is. Specifically, it could be of interest to investigate the case of the Sr-doped lanthanum manganite. Since the Sr-doped ma- terial has a stronger tendency to $d$-band formation, which works opposite to electron localization effects, it might be that such films could be grown under strain without structural changes even in the ultrathin film regime.

\section{ACKNOWLEDGMENT}

This work is part of the research program of the "Stichting voor Fundamenteel Onderzoek der Materie (FOM)," which is financially supported by NWO.
*Present address: Department of Applied Physics, Materials Science Centre and Netherlands Institute for Metals Research, University of Groningen, Nijenborgh 4, 9747 AG Groningen, The Netherlands.

${ }^{1}$ R. von Helmolt, J. Wecker, B. Holzapfel, L. Schultz, and K. Samwer, Phys. Rev. Lett. 71, 2331 (1993).

${ }^{2}$ E. Dagotto, T. Hotta, and A. Moreo, Phys. Rep. 344, 1 (2001).

${ }^{3}$ J. M. D. Coey, in Spin Electronics, edited by M. Ziese and M. J. Thornton, Lecture Notes in Physics Vol. 569 (Springer, New York, 2001).

${ }^{4}$ C. Zener, Phys. Rev. 82, 403 (1955); see also P. W. Anderson and H. Hasegawa, ibid. 100, 675 (1955).

${ }^{5}$ D. I. Khomskii and G. A. Sawatzky, Solid State Commun. 102, 87 (1997).

${ }^{6}$ R. J. Soulen, J. M. Byers, M. S. Osofsky, B. Nadgorny, T. Ambrose, S. F. Cheng, P. R. Broussard, C. T. Tanaka, J. Nowak, J. S. Moodera, A. Barry, and J. M. D. Coey, Science 282, 85 (1998).

${ }^{7}$ A. J. Millis, Nature (London) 392, 147 (1998).

${ }^{8}$ J. B. Goodenough and J. M. Longo, in Landolt-Börnstein, New Series Vol. 4a (Springer, New York, 1970).

${ }^{9}$ A. M. Glazer, Acta Crystallogr., Sect. B: Struct. Crystallogr. Cryst. Chem. B28, 3384 (1972).

${ }^{10}$ P. M. Woodward, Acta Crystallogr., Sect. B: Struct. Sci. B53, 32 (1997); B53, 44 (1997)

${ }^{11}$ H. Y. Hwang, S.-W. Cheong, P. G. Radaelli, M. Marezio, and B. Batlogg, Phys. Rev. Lett. 75, 914 (1995).

${ }^{12}$ Y. Tokura, Y. Tomioka, H. Kuwahara, A. Asamitsu, Y. Moritomo, and M. Kasai, J. Appl. Phys. 79, 5288 (1996).

${ }^{13}$ S.-W. Cheong and H. Y. Hwang, in Contribution to Colossal Magnetoresistance Oxides, Monographs in Condensed Matter Science, edited by Y. Tokura (Gordon \& Breach, London, 1999).

${ }^{14}$ A. Biswas, M. Rajeswari, R. C. Srivastava, Y. H. Li, T. Venkatesan, R. L. Greene, and A. J. Millis, Phys. Rev. B 61, 9665 (2000).

${ }^{15}$ A. Biswas, M. Rajeswari, R. C. Srivastava, T. Venkatesan, R. L.
Greene, Q. Lu, A. L. de Lozanne, and A. J. Millis, Phys. Rev. B 63, 184424 (2001).

${ }^{16}$ Z. Q. Yang, R. Hendrikx, J. Aarts, Y. Qin, and H. W. Zandbergen, Phys. Rev. B 67, 024408 (2003).

${ }^{17}$ J. Aarts, S. Freisem, R. Hendrikx, and H. W. Zandbergen, Appl. Phys. Lett. 72, 2975 (1998).

${ }^{18}$ H. W. Zandbergen, S. Freisem, T. Nojima, and J. Aarts, Phys. Rev. B 60, 10259 (1999).

${ }^{19}$ C. Traeholt, J. G. Wen, V. Svetchnikov, A. Delsing, and H. W. Zandbergen, Physica C 206, 318 (1993).

${ }^{20}$ Q. Huang, A. Santoro, J. W. Lynn, R. W. Erwin, J. A. Borchers, J. L. Peng, K. Ghosh and R. L. Greene, Phys. Rev. B 58, 2684 (1998).

${ }^{21}$ H. S. Wang, E. Wertz, Y. F. Hu, Q. Li, and D. G. Schlom, J. Appl. Phys. 87, 7409 (2000).

${ }^{22}$ S. Freisem, T. Nojima, R. W. A. Hendrikx, H. W. Zandbergen, and J. Aarts, Proc. SPIE 3481, 342 (1998); also S. Freisem, Ph.D. dissertation, Leiden University, 1999.

${ }^{23}$ R. A. Rao, D. Lavric, T. K. Nath, C. B. Eom, L. Wu, and F. Tsui, Appl. Phys. Lett. 73, 3294 (1998).

${ }^{24}$ H. W. Zandbergen and J. Jansen, Ultramicroscopy 80, 59 (1999).

${ }^{25}$ H. W. Zandbergen, J. Jansen, S. Freisem, T. Nojima, and J. Aarts, Philos. Mag. A 80, 337 (2000).

${ }^{26}$ M. Bibes, Ll. Balcells, S. Valencia, J. Fontcuberta, M. Wojcik, E. Jedryka, and S. Nadolski, Phys. Rev. Lett. 87, 067210 (2001).

${ }^{27}$ M. Bibes, Ll. Balcells, S. Valencia, S. Sena, B. Martinez, J. Fontcuberta, S. Nadolski, M. Wojcik, and E. Jedryka, J. Appl. Phys. 89, 6686 (2001).

${ }^{28}$ A. J. Millis, T. Darling, and A. Migliori, J. Appl. Phys. 83, 1588 (1998).

${ }^{29}$ Y. L. Qin, H. W. Zandbergen, Z. Q. Yang, and J. Aarts (unpublished).

${ }^{30}$ J. Klein, J. B. Philipp, G. Carbone, A. Vigliante, L. Alff, and R. Gross, Phys. Rev. B 66, 052414 (2002).

${ }^{31}$ Z. Fang, I. V. Solovyev, and K. Terakura, Phys. Rev. Lett. 84, 3169 (2000). 